\title{
Short-course hypofractionated radiochemotherapy for unresectable locally advanced cancer of the base of tongue: palliation only? A case report and short review of the literature
}

\author{
Maria-Aggeliki Kalogeridi, MD, PhD', Vassilios Kouloulias, MD, PhD², \\ Anna Zygogianni, MD, PhD ${ }^{1,3}$, George Kyrgias, MD, PhD ${ }^{1,3}$ \\ 'Department of Radiation Oncology, University Hospital of Larissa, Larissa; ${ }^{2} 2$ nd Department of Radiology-Radiotherapy Unit, \\ Faculty of Medicine, School of Health Sciences, Kapodistrian University of Athens, Athens; ${ }^{3}$ Department of Radiotherapy, \\ Faculty of Medicine, School of Health Sciences, University of Thessaly, Larissa, Greece
}

We present a case of unresectable cancer of the base of tongue treated with hypofractionated 3D conformal radiotherapy and concomitant chemotherapy. Based on the excellent tumour response in this radiotherapy regimen and international experience in short course treatments we shortly reviewed, we propose that this therapeutic approach could be considered in a curative setting for patients unsuitable for the a standard long course radiochemotherapy schedule.

Keywords: Head neck neoplasms, Base of tongue neoplasms, Hypofractionated radiotherapy, Chemoradiotherapy

\section{Introduction}

Randomised trials and meta-analyses have established a seven-week concomitant radiochemotherapy course as the standard radical treatment for patients with unresectable locally advanced head and neck cancer. However, a significant number of these patients are not suitable for this standard therapeutic approach. This includes patients with severe medical comorbidities who could not tolerate the toxicity associated with a long course of radiochemotherapy and patients in a poor general physical or mental condition. In addition, the standard approach is problematic for patients who live a long way from the treatment centre and who are required to make the journey on a daily basis for seven weeks. All these patients still require an effective treatment that can offer high rates of symptomatic palliation with acceptable toxicity, as approximately 50\% of untreated head and neck cancer patients will die within 4 months of their diagnosis. Although radiation oncologists have for a long time tried to identify the appropriate dose and fractionation for palliative head and neck cancer radiotherapy the optimal schedule has not been defined yet.

\section{Case Report}

A 58-year-old man with progressive difficulties in swallowing and articulation was referred to our department for radiotherapy of locally advanced squamous cell carcinoma of the base of tongue. The patient was a heavy smoker and had a history of drug abuse.

On physical examination, the patient had a lesion fixed to the right part of the base of tongue and ipsilateral palpable

Received 28 February 2014, Revised 12 May 2014, Accepted 27 May 2014.

Correspondence: George Kyrgias, MD, PhD, Department of Radiotherapy, Faculty of Medicine, School of Health Sciences, University of Thessaly, Biopolis, Larissa 41110, Thessaly, Greece. Tel: +30-2413-502050, E-mail: gkyrgias@gmail.com

(c) This is an Open Access article distributed under the terms of the Creative Commons Attribution Non-Commercial License (http://creativecommons.org/ licenses/by-nc/3.0/) which permits unrestricted non-commercial use, distribution, and reproduction in any medium, provided the original work is properly cited.

www.e-roj.org 

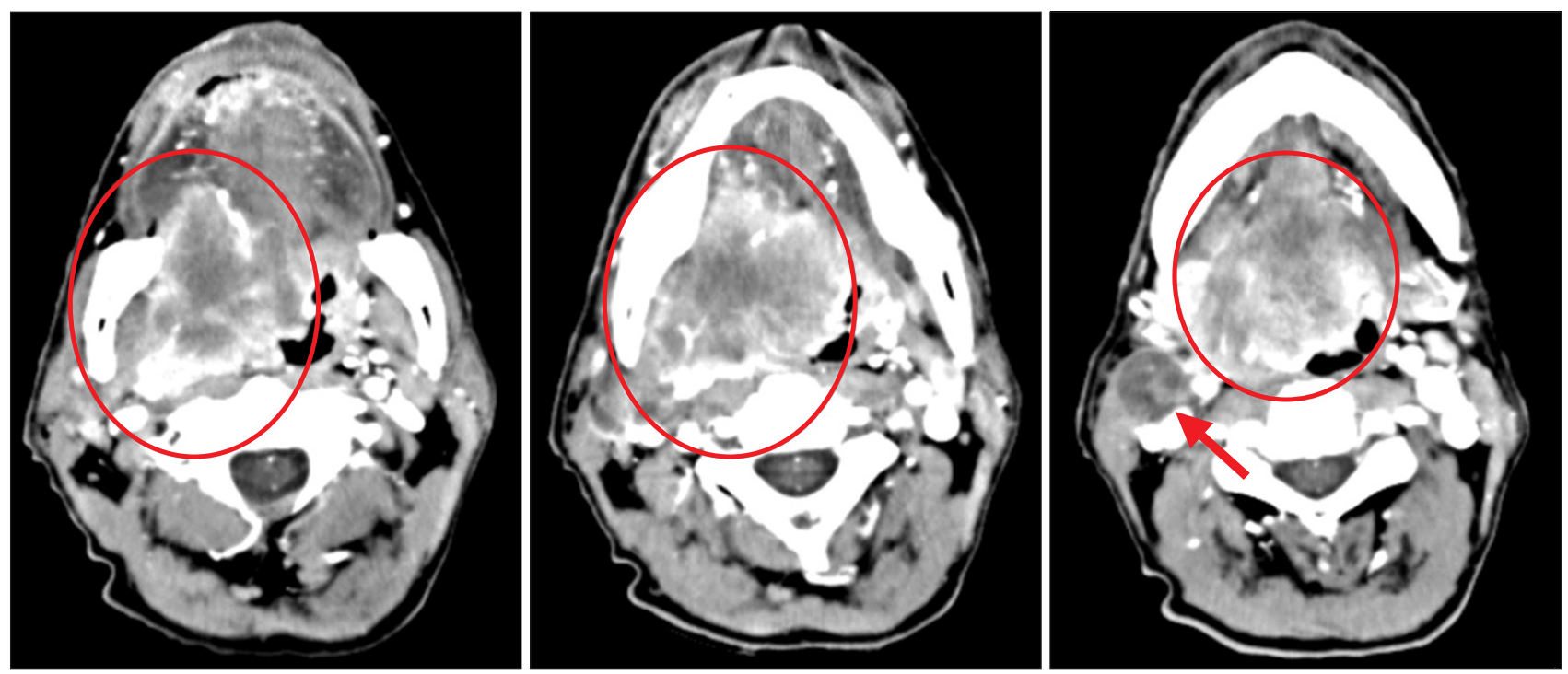

Fig. 1. Patient's computed tomography scan before treatment: the malignant growth is evident as a red-circled area. The red arrow indicates the right lymph node area involved by the disease.

posterior cervical lymph nodes. The CT scan revealed a $59 \times 43$ $\times 56 \mathrm{~mm}$ lesion of the right tongue base, invading the uvula and extending to the ipsilateral site of the epiglottis (redcircled area in Fig. 1) as well as a lymph node block of $35 \mathrm{~mm}$ diameter with central necrosis of the ipsilateral level IIA-IIB (red arrow in Fig. 1). Moreover, enlarged nodes of the ipsilateral level VA and nodes up to $16 \mathrm{~mm}$ of the contralateral levels IIB and upper VA have been found.

Positron emission tomography (PET) or PET-CT is a very important diagnostic procedure to be performed prior any kind of treatment in head and neck cancer. Indeed, PET-CT scan improves the accuracy of pre-treatment staging in terms of sensitivity and specificity and helps in better delineating the radiotherapy target. However, no PET-CT was performed to our patient due to the lack of PET-CT scanner in our center or nearby. Finally, our patient was human papillomavirus (HPV)negative although in international literature we found that $40 \%$ to $45 \%$ of oropharyngeal carcinomas are HPV-positive.

Since there was no evidence of metastatic disease according to chest computing tomography and liver ultrasound, the patient was staged as $\mathrm{T} 4 \mathrm{aN} 2 \mathrm{c}$ and the multidisciplinary meeting decided to offer him a course of concomitant radiochemotherapy. Given the refusal of the patient to attend the standard long course of radiotherapy and his relatively bad compliance due to drug abuse, we finally decided to offer him a course of hypofractionated radiotherapy with weekly cisplatin $40 \mathrm{mg} / \mathrm{m}^{2}$.
The patient was referred for dental assessment and placement of a percutaneous endoscopic gastrostomy tube in order to bypass feeding and eating difficulties during the radiotherapy course. Then he underwent a planning CT scan of the head and neck on the basis of which we prepared his treatment plan.

The patient was treated with hypofractionated 3D conformal radiotherapy. He received $30 \mathrm{~Gy}$ in 10 fractions in the primary tumour, neck and supraclavicular fossa and 15 Gy as a boost in the gross tumour volume, reaching a total dose of $45 \mathrm{~Gy}$, which is biologically equivalent to $54 \mathrm{~Gy}$ and clearly lower than the usual total therapeutic dose of $70 \mathrm{~Gy}$ given by conventional fractionation of $2 \mathrm{~Gy}$ per fraction.

The patient was assessed every week for a toxicity report and followed-up regularly both clinically and radiographically after the completion of radiotherapy.

At the end of the radiotherapy course there was significant clinical improvement with a substantial decrease in gross tumour volume and also in the level of pain, an increase in tongue mobility and improvement in articulation. Early toxicity was limited to grade II mucositis and skin toxicity no higher than grade II was observed. Overall treatment tolerance was excellent.

Twelve months after the completion of radiotherapy, the CT scan confirmed a near-total remission of primary lesion and a full regression of metastatic lymphnode, along with clinical improvement of the patient (Fig. 2). On further radiological and clinical follow-up there was no sign of local recurrence. 

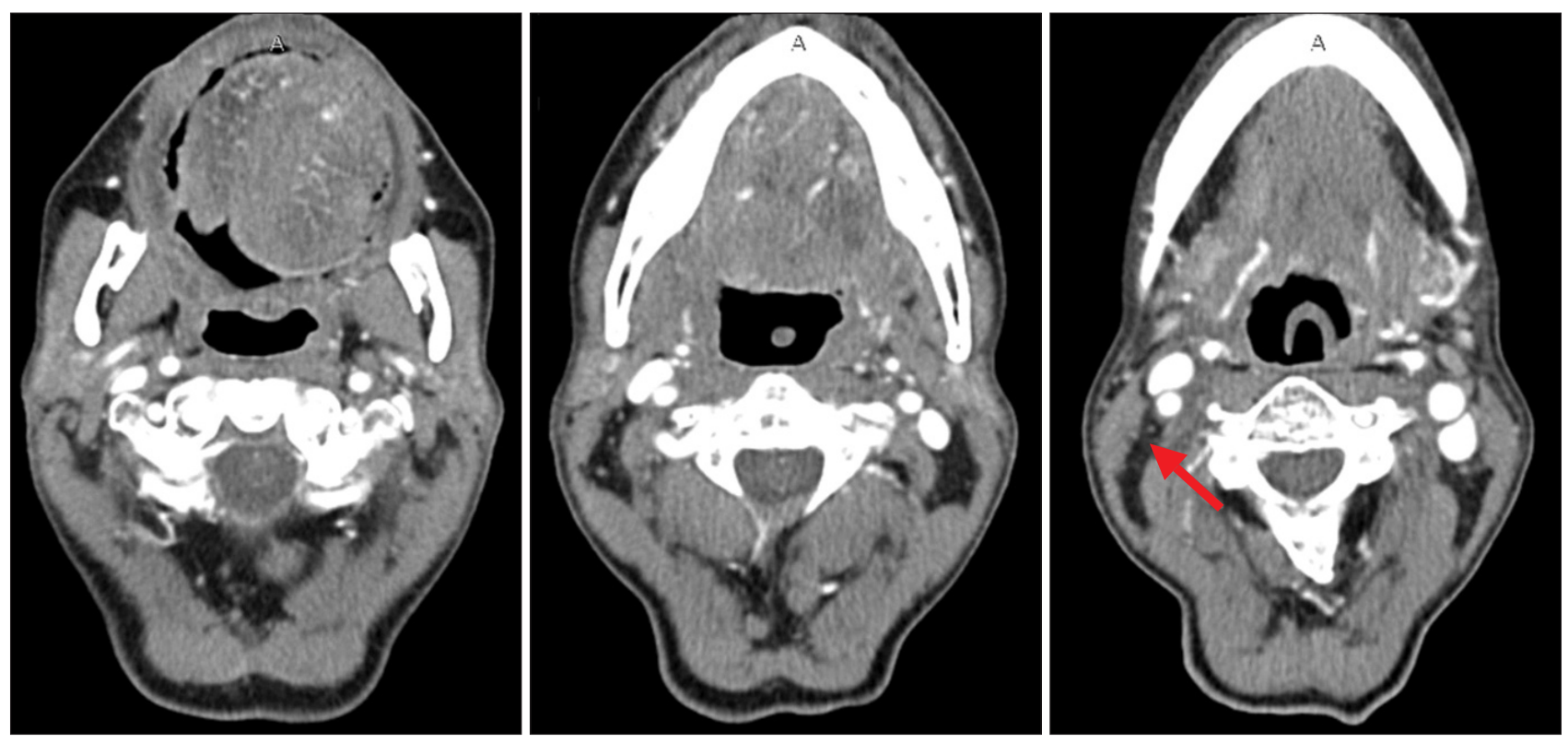

Fig. 2. Patient's computed tomography scan at twelve months after hypofractionated treatment. A near-total remission of primary disease and a full regression of the lymphnode metastasis can be seen (red arrow).

Eighteen months after the completion of radiotherapy the patient is free of recurrence with no swallowing or articulation difficulties and no signs of late skin toxicity.

\section{Discussion}

A study by Mohanti et al. [1] evaluated disease response, overall survival and symptom relief in a large cohort of patients receiving either palliative radiotherapy only (352 patients, $20 \mathrm{~Gy} / 5$ fractions) or up to $70 \mathrm{~Gy}$ (156 patients) if there was more than $50 \%$ objective regression at the tumour and nodal sites. More than $50 \%$ relief from distressing symptoms (pain, dysphagia, otalgia, respiratory distress, hoarseness, and cough) was achieved for $47 \%$ to $76 \%$ of patients receiving palliative radiotherapy. Median overall survival was 200 days. However, toxicity was rather high, with all patients experiencing dry desquamation skin erythema and patchy oropharyngeal mucositis at one month after palliative radiotherapy.

Another study by Agarwal et al. [2] evaluated the effectiveness and toxicity of a hypofractionated, palliative radiotherapy schedule consisting of $40 \mathrm{~Gy}$ in 16 fractions in 31/2 weeks. Patients with disease regression and acceptable acute toxicity were offered further dose escalation up to a maximum dose of $50 \mathrm{~Gy}$. Ten percent of the patients showed a complete response of $10 \%$ while $63 \%$ showed a partial response (6 to 8 weeks after completion of treatment) and one-year progression-free survival was $55.1 \%$. At completion of radiotherapy a $57 \%$ of patients presented $50 \%$ to $75 \%$ of symptomatic improvement while $17 \%$ presented $>75 \%$ symptomatic improvement as compared to baseline in pain, dysphagia, and hoarseness. However, $63 \%$ of the patients suffered from grade III mucositis and 54\% developed grade II xerostomia.

Recently, Monnier et al. [3] published a retrospective study of palliative hypofractionated radiation therapy with concurrent radiosensitising chemotherapy for 78 patients with advanced head and neck squamous cell carcinoma not eligible for conventional treatment. One-third of the patients had comorbid conditions and a performance status of 1 or 2. Radiotherapy was administered at 2 fractions of 3 Gy per day on days 1 and 3 during the first, third, fifth, and seventh weeks of treatment. Most of the patients received concurrent chemotherapy (mostly a combination of platinum and 5-fluorouracil). The overall incidence of grade 3-4 acute and late toxicity was low (4\% and 12\%, respectively). Forty one patients had a complete or partial response. Median overall survival was 12.9 months and the one-year progression-free survival rate was $51.5 \%$.

Another approach tested at the Yorkshire Cancer Centre for advanced head and neck cancer patients treated with palliative intent was a split course of radiation therapy with an initial 20 Gy in 5 fractions over 1 week, a 2-week gap, and then a further $20 \mathrm{~Gy}$ in 5 fractions over 1 week. The regimen proved quite 
effective, with symptomatic improvement reported in 79\% of patients and acceptable toxicity. Median overall survival and survival at 2 years was 9 months and 25\%, respectively [4].

Regarding the issue of toxicity, Zygogianni et al. [5] in their study on patients suffering from head and neck cancer and treated with a hypofractionated radiotherapy regimen demonstrated that the administration of oral glutamine reduced down to $10 \%$ the grade II and eliminated the grade III acute oral toxicity.

In conclusion, given the encouraging results of hypofractionated radiochemotherapy for patients with locally advanced head and neck cancer in palliative treatment, this therapeutic approach could be also considered in a curative setting for highly selected patients unsuitable for the standard long course radiochemotherapy schedule. Obviously, further clinical studies are needed to meet an evidence-based certainty on this issue.

\section{Conflict of Interest}

No potential conflict of interest relevant to this article was reported.

\section{References}

1. Mohanti BK, Umapathy H, Bahadur S, Thakar A, Pathy S. Short course palliative radiotherapy of 20 Gy in 5 fractions for advanced and incurable head and neck cancer: AlIMS study. Radiother Oncol 2004;71:275-80.

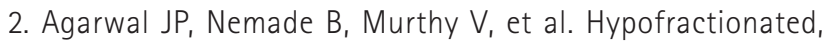
palliative radiotherapy for advanced head and neck cancer Radiother Oncol 2008;89:51-6.

3. Monnier L, Touboul E, Durdux C, Lang P, St Guily JL, Huguet F. Hypofractionated palliative radiotherapy for advanced head and neck cancer: the IHF2SO regimen. Head Neck 2013;35: 1683-8.

4. Kancherla KN, Oksuz DC, Prestwich RJ, et al. The role of splitcourse hypofractionated palliative radiotherapy in head and neck cancer. Clin Oncol (R Coll Radiol) 2011;23:141-8.

5. Zygogianni A, Kyrgias G, Kouvaris J, et al. Impact of acute radiation induced toxicity of glutamine administration in several hypofractionated irradiation schedules for head and neck carcinoma. Head Neck Oncol 2012;4:86. 